\title{
FOTOGRAMETRIA ESFÉRICA: UMA TÉCNICA DE BAIXO CUSTO PARA DOCUMENTAÇÃO ARQUITETÔNICA
}

\author{
Spherical Photogrammetry: a low-cost technique for architectural documentation
}

\author{
Andrea Verri Bastian \\ LCAD | Faculdade de Arquitetura | UFBA, Brasil. \\ avbastian@uol.com.br
}

\begin{abstract}
This paper reports the use of spherical photogrammetry used for the survey and documentation of buildings and developed at the Università Politecnica delle Marche, Ancona, Italy, the engineer and professor Gabriele Fangi. The paper presents and discusses the concepts and processes involved in this technique, as well as its use to obtain the main facade of the refund drawing the Church of St. Dominic, pointing its main advantages (such as low-cost technique) and disadvantages, as well as the results obtained when compared to traditional techniques photogrammetric.
\end{abstract}

Keywords: Heritage documentation. Digital Photogrammetry. Spherical Photogrammetry. Image based techniques for digital documentation.

\section{Introdução}

Este trabalho é resultado da dissertação de mestrado intitulada "Métodos e técnicas de baixo custo para levantamento de sítios históricos", apresentada ao Programa de Pós-graduação em Arquitetura e Urbanismo da Faculdade de Arquitetura da UFBA. A pesquisa teve como parte dos objetivos estudar e comparar técnicas digitais de baixo custo para o levantamento de edificações, através da captura e processamento dos dados, por meio de métodos fotogramétricos.

Com os avanços alcançados pelas tecnologias digitais, a fotogrametria digital terrestre tornou-se um método eficiente e econômico, sendo utilizada como técnica regular para o levantamento arquitetônico em vários países da Europa. Como se pode constatar em trabalhos publicados nos anais da CIPA Heritage Documentation e International Society for Photogrammetry and Remote Sensing (ISPRS), esta técnica é utilizada na maioria dos trabalhos publicados, o que comprova as suas vantagens para documentação do patrimônio histórico.

Assim, uma das etapas da pesquisa consistiu na documentação, de forma experimental, da fachada principal da Igreja de São Domingos localizada no centro histórico de Salvador, no estado da Bahia, através da restituição por meio da técnica denominada fotogrametria esférica.

\section{FOTOGRAMETRIA ESFÉRICA}

Segundo Fangi e colaboradores (2013), as imagens panorâmicas estão sendo cada vez mais empregadas em documentação do patrimônio cultural, principalmente quando integradas a sistemas de varredura a laser. Diferentes técnicas podem ser aplicadas para a geração de imagens panorâmicas como câmeras digitais rotativas (imagens gigapixel), conjunto de câmeras como as utilizadas pelo Google Street View e Cyclomedia. Pesquisas realizadas com esses sistemas, quando considerada a calibração da câmera e a orientação das imagens, apontam seu alto custo como desvantagem em comparação às técnicas de obtenção de panoramas por meio de costura de imagens.

Desenvolvida na década de 1990, por Szeliskie e Shum, com o intuito de melhorar a baixa resolução das imagens digitais, a técnica de costura de imagens oferece a possibilidade de obtenção de panoramas pela fusão de imagens planas (mosaicos) que se sobrepõem parcialmente. Apresenta como vantagens a exigência de um número menor de imagens; um campo de visão maior e com melhor resolução do que uma imagem única (mesmo para um panorama parcial), o que possibilita uma melhor compreensão do objeto. Além disso, a captura das imagens pode ser feita com câmeras nãométricas colocadas em um tripé com cabeça esférica (três graus de liberdade), de modo que o centro de projeções e a distância focal permaneçam os mesmos durante toda a fase de tomada fotográfica (WAHBEH, 2011).

A técnica fotogramétrica baseada em panoramas esféricos, chamada de Fotogrametria Esférica segundo D'Annibale e colaboradores (2011), é uma técnica desenvolvida na Università Politecnica delle Marche, Ancona, Itália, pelo engenheiro e professor Gabriele Fangi. Essa técnica baseiase em um tipo de projeção cartográfica onde as imagens são capturadas a partir de um único ponto (estação), de modo a cobrir total $\left(360^{\circ}\right.$ sólidos) ou parcialmente o seu entorno, e projetadas em uma esfera virtual.

Para Fangi (2013), as vantagens no emprego da Fotogrametria Esférica são muitas, desde a velocidade para aquisição das imagens fotográficas, a cobertura de até 360ำ (ângulo de varredura), a alta resolução das imagens panorâmicas, a integralidade da documentação, até a ausência de distorções de perspectiva, que são estimadas e corrigidas pelo próprio "software de costura". 
$\mathrm{Na}$ Fotogrametria Esférica, as fotografias são inseridas em um software comercial (como PTGui), são "costuradas" e gerados os panoramas. Para que esses panoramas possam ser utilizados para se obter informações métricas sobre o objeto, devem-se cumprir certas exigências geométricas rígidas como: a coincidência do centro de projeção das imagens; e a compensação tanto da distorção radial quanto dos deslocamentos residual, horizontal e vertical. Assim, a imagem final é gerada quase sem distorção.

Após a costura, os panoramas são mapeados e projetados em uma esfera virtual de raio $R$ igual à distância focal da câmera utilizada, por meio da projeção equiretangular. Nessa projeção, os meridianos e paralelos são representados por linhas retas verticais e horizontais, igualmente espaçadas, e os dois polos por linhas retas de comprimento igual ao do equador.

Para que sejam obtidos dados tridimensionais de objeto é necessário que se tenha mais de um panorama e esses devem ter definidos seu posicionamento e rotação (orientação). O processo inicia-se com a formação do modelo, utilizando a condição de coplanaridade, com apenas duas esferas virtuais; com isto, são determinados seis parâmetros de orientação por panorama. Assim, os modelos são referenciados dois a dois, e posteriormente "ligados" entre si, de maneira similar à fotogrametria clássica. Maiores detalhes sobre os conceitos envolvidos podem ser vistos em Fangi (2007; 2009; 2010; 2013), Fangi e Nardinocchi, (2013).

O fluxograma de trabalho em Fotogrametria Esférica pode ser resumido conforme o Figura 1 a seguir.

Após a orientação dos panoramas já é possível realizar a restituição fotogramétrica para geração dos modelos em wireframe (estrutura de arame).

Essas etapas de orientação dos panoramas são realizadas através do Pacote Sphera, descrito na Seção a seguir.

\section{Pacote Sphera}

O pacote Sphera é composto por três programas ou módulos a saber:

PointsRecord - por meio dele é criado um arquivo texto que contém os dados que representam as coordenadas dos pontos da imagem e um arquivo de linhas que serão empregadas na restituição. Nesse programa são carregados os panoramas criados no software de "costura" PTGui e localizados os posicionamentos das estações no momento da captura fotográfica. Após a localização das estações em todos os panoramas é feita a marcação dos pontos homólogos, sendo necessários pelo menos quatro pontos em dois panoramas para que a porcentagem de erro seja menor;

Sphera - utilizado para orientar os panoramas, o módulo gera um arquivo de dados, contendo os parâmetros e posicionamento das coordenadas que serão usadas no ajuste dos feixes perspectivos. Nesta etapa é necessário fornecer as informações referentes ao comprimento e largura dos panoramas, como também, a lista de coordenadas conhecidas tomadas em campo. Esses dados serão empregados para orientação absoluta e determinação da escala do modelo; após esse ajuste, o programa fornece três arquivos, contendo: os erros residuais para a conferência da qualidade do processo, em que o valor do parâmetro SIGMA NAUGHT deve ser o menor possível; o modelo gerado (arquivo DXF); e os parâmetros de orientação dos panoramas, as coordenadas relativas do centro da esfera e os três ângulos de orientação.

Panoplan - utilizado para restituição de detalhes contidos em uma superfície plana, a partir de um único panorama. Utilizando o PointRecords, primeiramente é necessária a criação de um arquivo identificando o plano de referência, definido a partir de pelo menos três pontos não alinhados;

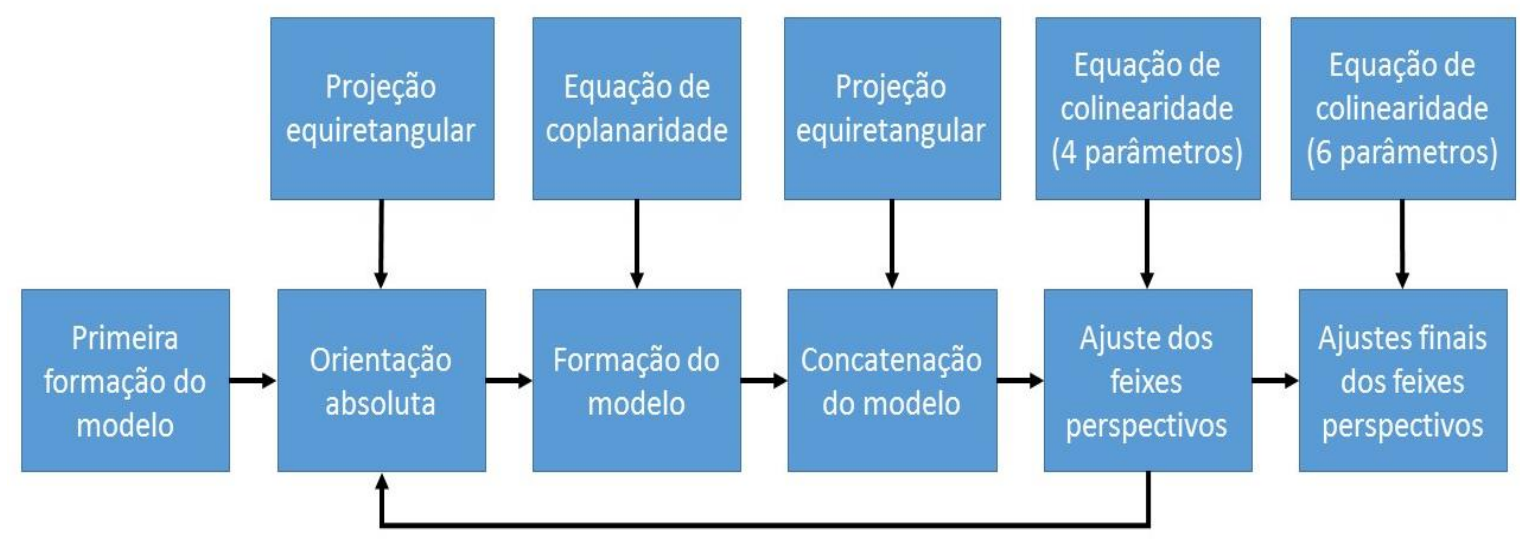

Figura 1 - Fluxograma de trabalho em Fotogrametria Esférica. Fonte: adaptado de FANGI; NARDINOCCHI, 2013.

o segundo passo é desenho do detalhe utilizando o panorama mais frontal possível, também no PointRecords. 
De posse desses dois arquivos o programa Panoplan gera um terceiro arquivo no formato DXF, contendo o plano de referência com o desenho do detalhe.

\section{Estudo de caso}

A fase experimental da pesquisa consistiu na restituição da fachada principal da Igreja de São Domingos.

\section{Igreja da Ordem Terceira de São Domingos}

A Igreja de São Domingos, situada no Centro Histórico de Salvador, tem sua fachada principal voltada para o Terreiro de Jesus, onde se localizam além da igreja do antigo Colégio de Jesus, a atual Catedral, a lgreja de São Pedro dos Clérigos e sobrados; fazendo parte de um conjunto de construções barrocas terminadas em pleno século XIX (TELLES, 2007, p. 89).

Foi a primeira igreja da Bahia a adotar o partido arquitetônico de três corpos de edificações separados por corredores longitudinais; segundo O IPHAN é uma construção de "[...] excepcional valor arquitetônico que compreende, além da igreja, dois corpos de edificações laterais abrigando as instalações da Ordem Terceira". Ainda, de acordo com IPHAN, "[...] a igreja apresenta planta típica das igrejas de irmandade do começo do século XVIII, com corredores laterais e tribunas superpostas. A fachada rococó tem uma torre terminada em bulbo. A talha neoclássica de Antonio Mendes de Silva, substitui a primitiva talha barroca. A pintura do teto da nave, atribuída a José Joaquim da Rocha é de concepção ilusionista barroca, lançada na Itália em 1694 por Andrea Pozzo, na igreja de Santo Inácio em Roma e introduzida no Brasil no final da década de 1720/1730" (IPHAN, 2015, p. 23).

Sua construção foi iniciada por volta de 1731 e o tombamento da Igreja foi realizado em 1938.

\section{Restituição por meio de fotogrametria esférica}

Para a restituição da fachada, foram adotados os seguintes procedimentos:

- tomada fotográfica - foi realizada com a câmera Canon EOS 60D, com resolução de 18 megapixeis, objetiva com distância focal $28 \mathrm{~mm}$, em um tripé com cabeça esférica de modo que o centro de projeções permanecesse fixo durante toda a fase de tomada e que todo o objeto fosse registrado. Para o estudo em questão, foram capturadas 144 fotografias (com sobreposição de 15 a $20 \%$ ) a partir de 7 estações $^{1}$, correspondentes a pontos de vista diferentes (Figura 2);

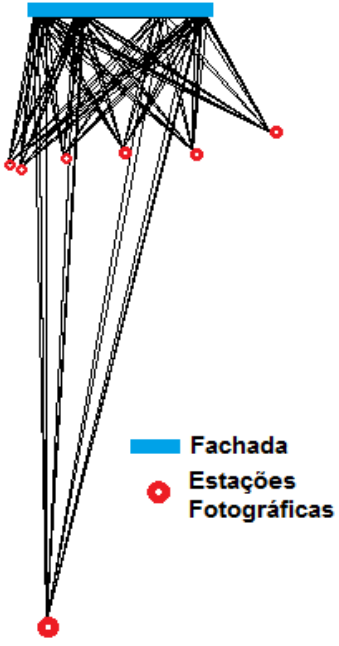

Figura 2 - Fotogrametria esférica: posicionamento das estações fotográficas. Fonte: Andrea Bastian, 2014.

- criação dos panoramas - as fotografias foram inseridas no software de costura PtGui e gerados os panoramas (Figura 3) com uma sobreposição média de $30 \%$.

\footnotetext{
1 O número de estações é definido pelo posicionamento da câmera no momento das tomadas fotográficas necessárias para a cobertura adequada da fachada.
} 


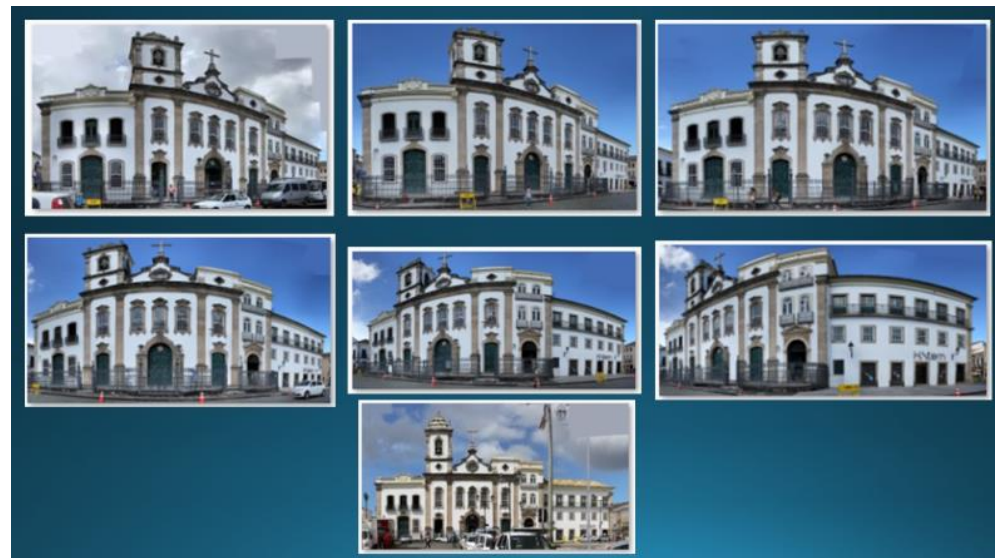

Figura 3 - Fotogrametria esférica: imagens panorâmicas. Fonte: Gabriele

Fangi, 2013.

- uso do PointsRecord - os panoramas foram inseridos no programa, marcados os pontos relativos às estações fotográficas (7 estações) e algumas feições de interesse nas sete imagens.

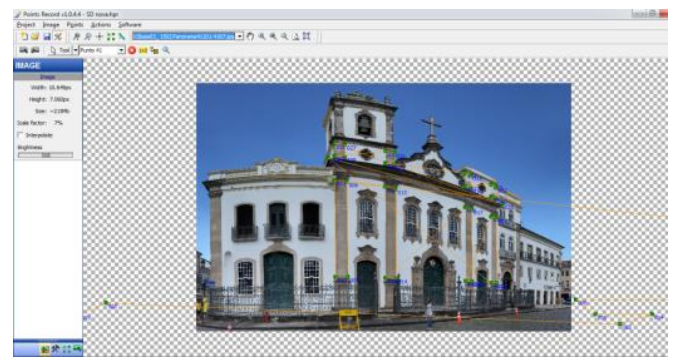

Figura 4 - Fotogrametria esférica: captura de tela do PointsRecord Fonte: Andrea Bastian, 2014.

- uso do Sphera - a cada dois panoramas, esse módulo executou o ajuste dos feixes perspectivos. O processo fornece o modelo geométrico "aramado" em um arquivo em formato DXF.

- restituição - a restituição dos pontos foi realizada por partes, através do módulo PANO. Para tando, primeiramente foi utilizado o PointsRecords, marcados os pontos homólogos em pelo menos três imagens, executado o PANO e assim gerado um outro arquivo DXF para inserção, como bloco, no primeiro modelo obtido. Esse processo foi realizado várias vezes até que as principais feições completassem o modelo da fachada; e
- monorrestituição - para a restituição de detalhes pertencentes a um único plano.

A Figura 5 apresenta o desenho de restituição de um detalhe da fachada da Igreja de São Domingos.

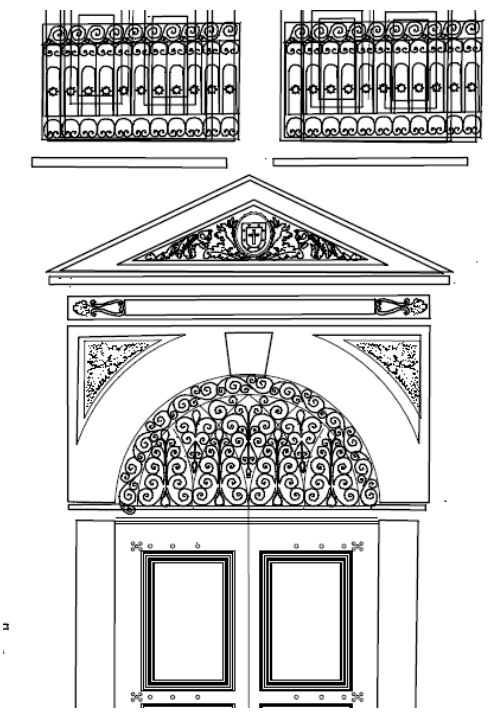

Figura 5 - Detalhe do desenho de restituição da fachada principal da Igreja de São Domingos. Fonte: Andrea Bastian, 2015.

Já a Figura 6 mostra o desenho de restituição da fachada da Igreja, obtido a partir do processo descrito; para obtenção deste desenho parcial, foram gerados 92 arquivos DXF e inseridos os blocos no modelo inicial. 


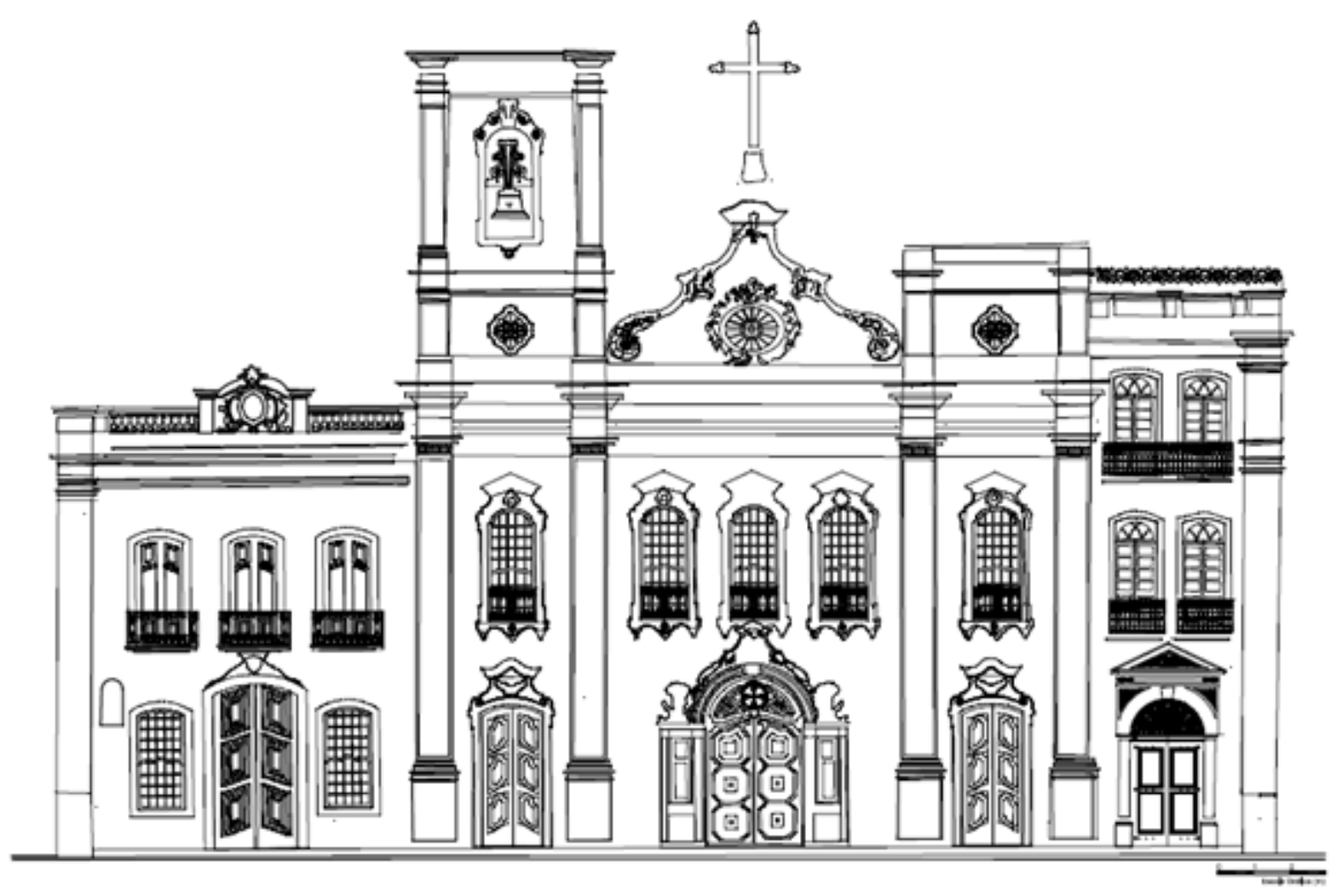

Figura 6 - Desenho da restituição (parcial) da fachada principal da Igreja de São Domingos. Fonte: Andrea Bastian, 2015.

\section{Restituição por meio de fotogrametria digital "tradicional"}

A fim de comparar processos e produtos gerados, a mesma fachada foi restituída utilizando fotogrametria digital "tradicional", aqui neste trabalho referindo-se à técnica de restituição fotogramétrica baseada em fotos convergentes, por meio da ferramenta PhotoModeler Scanner, versão 2012. Por se tratar de uma técnica bastante difundida, assim como o programa utilizado, os conceitos envolvidos não serão discutidos. Maiores detalhes podem ser vistos em Bastian (2015) e Groetelaars (2004) entre outros.

Na Figura 7 encontra-se a síntese do fluxo de trabalho para utilização da ferramenta PhotoModeler Scanner.

Para o levantamento da fachada do edifício, foram necessárias duas etapas em campo. A primeira etapa consistiu na aquisição de medidas, por medição direta, efetuada com trena de aço, de pelo menos duas dimensões registradas em croqui esquemático; valores esses utilizados, posteriormente, na fase de gabinete para a correção da escala do modelo. Num segundo momento, foi realizado o levantamento fotográfico.

A captura fotográfica foi feita com câmera Nikon D300, resolução de 12,3 megapixeis, objetiva com distância focal $12 \mathrm{~mm}$ e $20 \mathrm{~mm}$. Para o estudo em questão foram capturadas 46 fotografias. Entretanto, apenas nove foram suficientes para a restituição da fachada.
$\mathrm{Na}$ fase executada em gabinete, os dados foram processados, sendo a fachada restituída e gerada a ortofoto. Num segundo momento obtido o desenho de restituição por meio da vetorização interativa.

A Figura 8 apresenta o detalhe do desenho de restituição da fachada da Igreja de São Domingos.

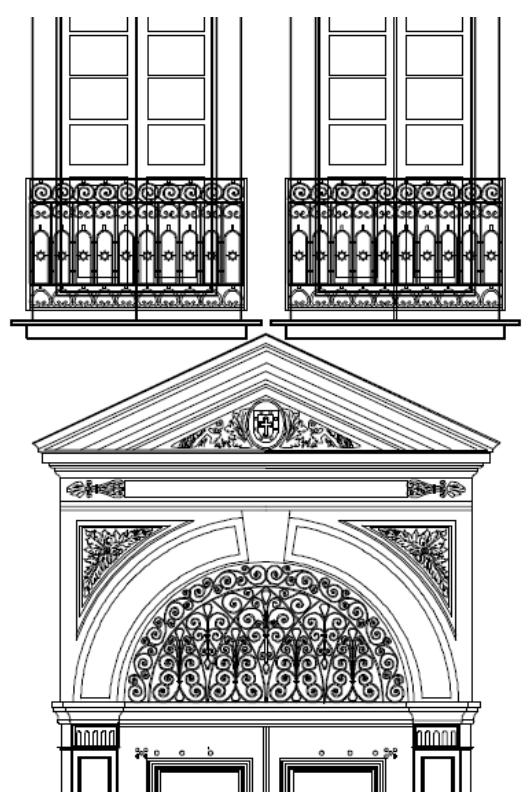

Figura 8 - Detalhe do desenho de restituição da fachada principal da Igreja de São Domingos. Fonte: Andrea Bastian, 2015. 


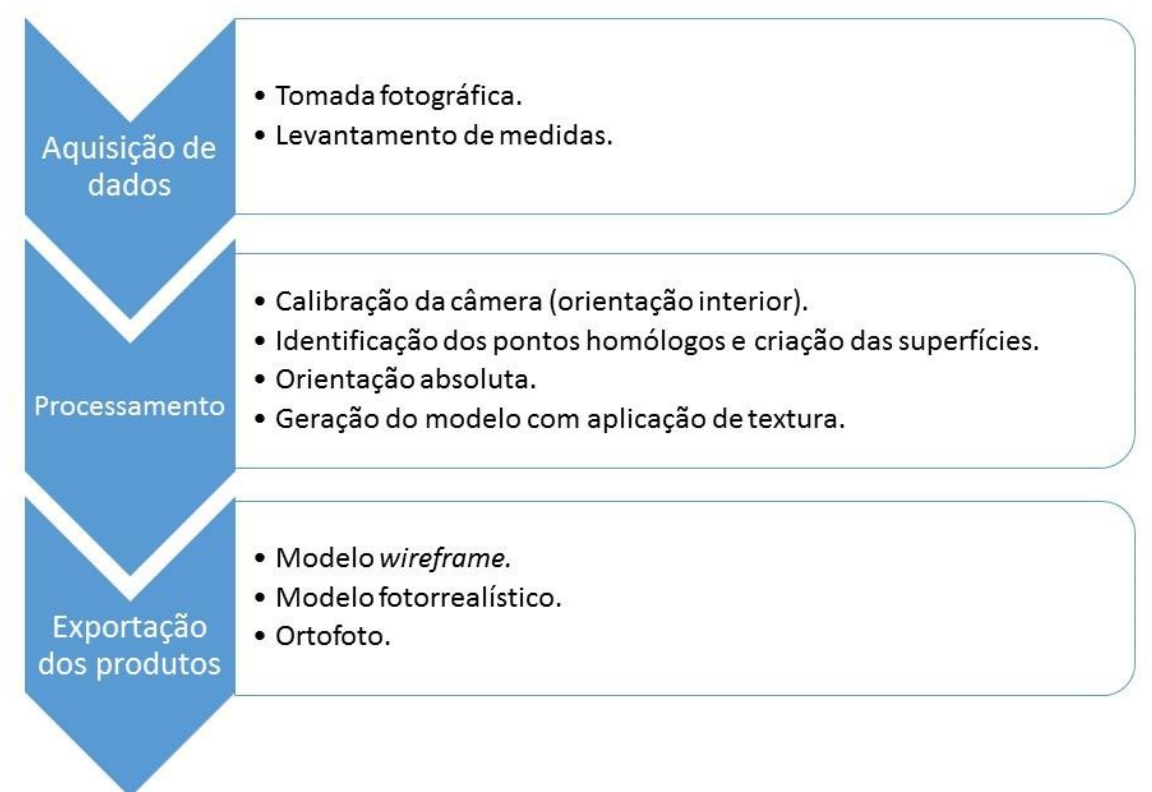

Figura 7 - Síntese do fluxo de trabalho no programa PhotoModeler. Fonte: Andrea Bastian, 2014.

A Figura 9 mostra o desenho de restituição da fachada da Igreja, obtido a partir do processo descrito.

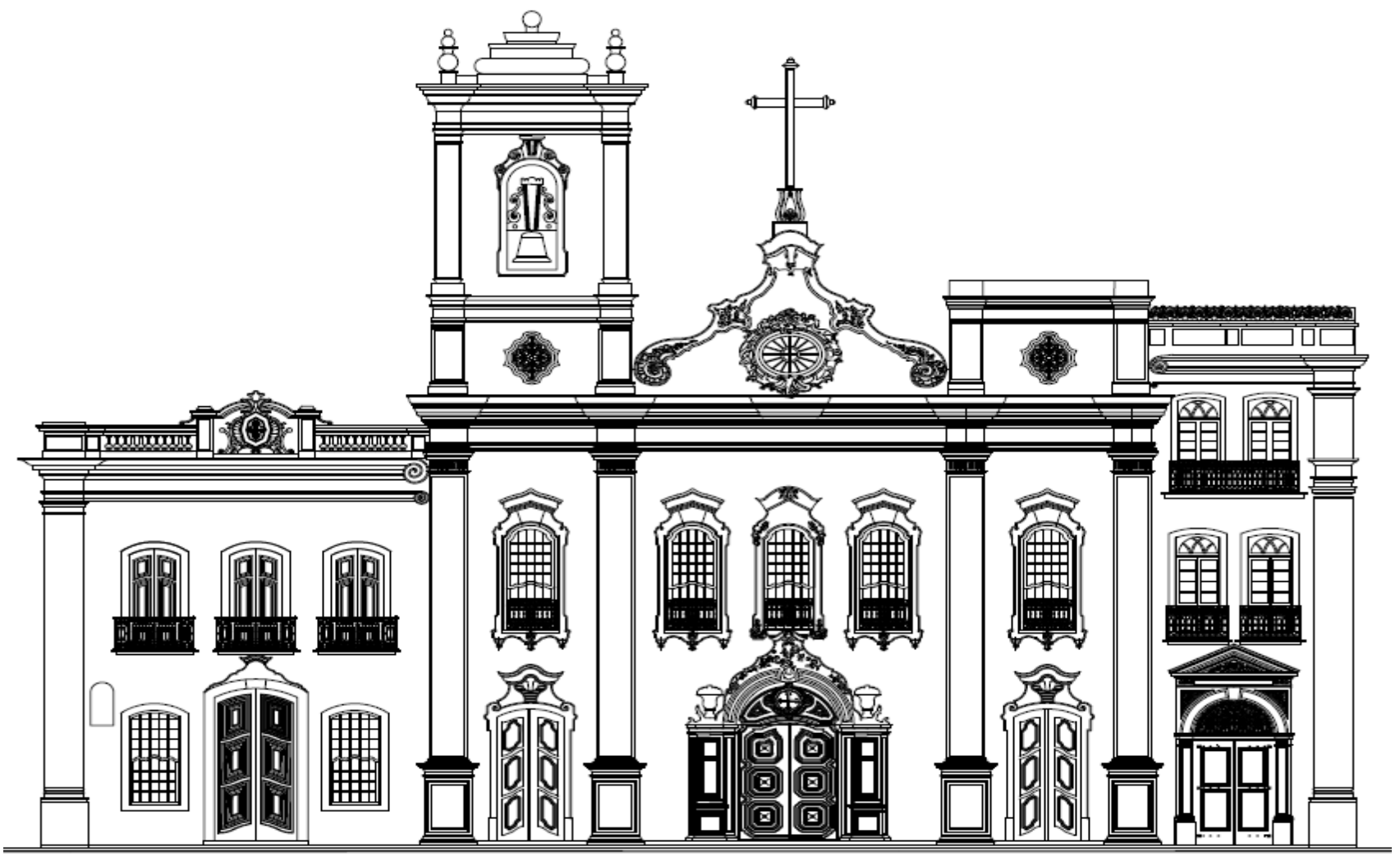

Figura 9 - Desenho da restituição (parcial) da fachada principal da lgreja de São Domingos. Fonte: Andrea Bastian, 2015. 


\section{Considerações finais}

Os levantamentos aqui apresentados, por meio da fotogrametria esférica, tiveram como objetivo apropriar-se da ferramenta e explorá-la, estudando suas possibilidades. Assim, pode-se verificar que existe maior facilidade na captura dos detalhes das edificações, uma vez que os panoramas apresentam resolução bastante alta, embora o processo de restituição seja mais lento. Em que pese a obtenção de modelos wireframe, outro ponto importante é a obtenção de arquivos no formato DXF, facilitando o processo de desenho, pois elimina a etapa de vetorização interativa realizada sobre a ortofoto gerada, como no caso de uso do PhotoModeler.

Outro ponto importante é fornecer como subprodutos os próprios panoramas, que registram com precisão a edificação e podem ser utilizados em outras aplicações.

Pode-se considerar como um ponto crítico a falta de um programa único, em que todas as fases do processo possam ser realizadas de modo mais automatizado, gerando um volume menor de arquivos. Enquanto o programa PhotoModeler fornece a ortofoto, o modelo wireframe obtido na fotogrametria esférica deve ser

FANGI, G. (2007). The multi-image spherical panoramas as a tool for architectural survey. INTERNATIONAL CIPA SYMPOSIUM, 21.

FANGI, G. (2009). Further Developments of the SPHERICAL photogrammetry for cultural heritage. INTERNATIONAL CIPA SYMPOSIUM, 22.

FANGI, G. (2009). Multiscale Multiresolution Spherical Photogrammetry with long focal lenses for Architectural Surveys. INTERNATIONAL ARCHIVES OF PHOTOGRAMMETRY, REMOTE SENSING AND SPATIAL INFORMATION SCIENCES, 38.

FANGI, G. (2013). Spherical photogrammetry for cultural heritage metric documentation: a critical examen six years after the beginning. BUILT HERITAGE MONITORING CONSERVATION MANAGEMENT, 1.

FANGI, G.; NARDINOCCHI C. (2013). Photogrammetric processing of spherical panoramas. The Photogrammetric Record: The Remote Sensing and Photogrammetry Society and John Wiley \& Sons Ltd, n. 28, p. 293-311.<doi: 10.1111/phor.12031>.

GROETELAARS, N. J. (2004). Um Estudo da Fotogrametria Digital na documentação de formas arquitetônicas e urbanas. 2004. (Dissertação de mestrado), Universidade Federal da Bahia.

INSTITUTO DO PATRIMÔIO HISTÓRICO E ARTISTICO NACIONAL - IPHAN (2015). Proposta de inscrição na Lista do Patrimônio Mundial da UNESCO (pp. 78).

WAHBEH, W. (2011). Architectural Digital Photogrammetry Panoramic Image-Based Interactive Modelling (Tesi Dottorato importado em outras ferramentas de modelagem, servindo como referência para a criação de superfícies e aplicação da textura das imagens, para que se possa obter modelos mais realísticos.

Ainda, a identificação do posicionamento das estações fotográficas e a orientação dos panoramas mostraram-se os maiores gargalos no processo de entendimento do uso dos programas.

\section{Agradecimentos}

Ao professor Gabrielle Fangi pela cessão do Pacote Sphera, pela geração dos panoramas utilizados na pesquisa, e sobretudo pela paciente orientação.

\section{Referências}

BASTIAN, A. V. (2015). Métodos e técnicas de baixo custo para levantamento métrico de sítios históricos. (Dissertação de mestrado), Universidade Federal da Bahia.

D'ANNIBALE, E.; PIERMATTEI, L.; FANGI, G. (2011). The Spherical Photogrammetry as Emergency Photogrammetry. INTERNATIONAL CIPA SYMPOSIUM, 23.

in Storia, Disegno e Restauro dell'Architettura), Università degli Studi di Roma "La Sapienza".

SZELISKI, R.; SHUM, H. (1997). Creating full view panoramic image mosaics and environment maps. CONFERENCE ON COMPUTER GRAPHICS AND INTERACTIVE TECHNIQUES, 24.

TELLES, A. C. (2007). Atlas dos Monumentos Históricos e Artísticos do Brasil. Rio de Janeiro: FENAME/DAC. 
SIGraDi 2016, XX Congress of the Iberoamerican Society of Digital Graphics 9-11, November, 2016 - Buenos Aires, Argentina 